\title{
Selecting, adapting, and sustaining programs in health care systems
}

\author{
This article was published in the following Dove Press journal: \\ Journal of Multidisciplinary Healthcare \\ 16 April 2015 \\ Number of times this article has been viewed
}

\author{
Leah L Zullig',2 \\ Hayden B Bosworth ${ }^{1-4}$ \\ 'Center for Health Services Research \\ in Primary Care, Durham Veterans \\ Affairs Medical Center, Durham, NC, \\ USA; ${ }^{2}$ Department of Medicine, Duke \\ University Medical Center, Durham, \\ NC, USA; ${ }^{3}$ School of Nursing, \\ ${ }^{4}$ Department of Psychiatry and \\ Behavioral Sciences, Duke University, \\ Durham, NC, USA
}

\begin{abstract}
Practitioners and researchers often design behavioral programs that are effective for a specific population or problem. Despite their success in a controlled setting, relatively few programs are scaled up and implemented in health care systems. Planning for scale-up is a critical, yet often overlooked, element in the process of program design. Equally as important is understanding how to select a program that has already been developed, and adapt and implement the program to meet specific organizational goals. This adaptation and implementation requires attention to organizational goals, available resources, and program cost. We assert that translational behavioral medicine necessitates expanding successful programs beyond a standalone research study. This paper describes key factors to consider when selecting, adapting, and sustaining programs for scale-up in large health care systems and applies the Knowledge to Action (KTA) Framework to a case study, illustrating knowledge creation and an action cycle of implementation and evaluation activities.
\end{abstract}

Keywords: program sustainability, diffusion of innovation, information dissemination, health services research, intervention studies

\section{Introduction}

Clinicians and health services researchers often design programs to address specific health problems. Despite evidence that a program may be effective, many programs become one-time, time-limited interventions. Even among interventions that are disseminated, the transfer of research findings into clinical practice is often a slow and haphazard process. ${ }^{1,2}$ This minimal translation into practice may be attributed to lack of planning for future scalability. Scaling up a successful program and broadly implementing it (or translating knowledge into action), perhaps in the context of a health care system, could maximize potential impacts on individual and population health.

We assert that translational behavioral medicine necessitates expanding successful programs beyond a stand-alone research study. Innovative programs must be intentionally developed with future scale-up and implementation potential in heterogeneous organizations. We discuss developing scalable programs, selecting an existing program that meets an organization's needs, adapting an existing program to fit the unique organizational culture and patient populations, and sustaining interventions long-term. Once an intervention has been developed, translating it into the field (eg, turning knowledge into action) is a critical, yet often overlooked, step. ${ }^{1}$ We present an antihypertensive medication adherence and patient self-monitoring intervention as a case study because it represents two complex, but common, required behaviors. 


\section{Theoretical framework}

The Knowledge to Action (KTA) Framework was developed based on a review of 31 planned action theories with the goal of providing a framework for thinking about the process and integration of knowledge creation and knowledge application. ${ }^{1,3}$ Stated differently, the theory outlines a process for exchanging knowledge between relevant stakeholders in a way that results in action. ${ }^{1}$ The KTA Framework is comprised of two components: knowledge creation and an action cycle. ${ }^{3}$ Each component has multiple phases. For example, knowledge creation encompasses knowledge inquiry, synthesis, and products/tools. ${ }^{1,3,4} \mathrm{~A}$ recent literature review concluded that the KTA Framework is used in practice; many more studies have applied the action cycle rather than integrating the framework in its entirety. ${ }^{3}$ In the context of the KTA Framework, we present a case study demonstrating how it is possible to bridge the gap between a research intervention study (ie, knowledge creation) and action (ie, implementation in a health care system).

\section{Scaling interventions}

Scalability involves expanding a program that has been demonstrated as being efficacious on a controlled, small scale and implementing it under real world conditions with the goal of reaching a larger population. ${ }^{5}$ The potential for scalability is important to consider when developing a new program or selecting existing programs for broader implementation. In making the transition to a broad rollout, it is critical to first assess whether the program is worthy of scaling up; how effective is it at achieving the targeted behavioral change?
There may be problems with adopting an intervention before it has been clearly demonstrated to be advantageous for patients. ${ }^{1}$ If an intervention is adopted prior to its benefits being verified, it is possible that patients may be exposed to ineffective or potentially harmful treatments. ${ }^{1,6}$ Even safe and effective interventions may require modification for scale-up. Most effectiveness trials involve samples of 300-600 people. What needs to be altered when scaling a program for 3,000 or 6,000 individuals? A program must not only accomplish the desired behavioral change, such as improving medication adherence, but preferably that change must also be maintained within an individual over time (Table 1).

Next, it is important to consider the feasibility of scaling the program, given the resources required to implement and sustain it. These resources could include human, organizational, and technological resources as well as physical space. Contextual factors, such as the organization's readiness to change, must also be taken into account. To ensure successful scale-up, addressing both resource needs and contextual factors during the planning stages of the scale-up process is imperative.

Cost is arguably one of the most important factors to consider when scaling-up a program, yet many programs fail to adequately evaluate implementation cost. What are the per patient intervention costs? Is the program cost-effective? As the intervention is scaled up, how do costs change? Even if the program is more cost-effective than standard clinical care, the health care system must be able to afford it. Finally, there must be a program evaluation plan. Evaluating the program must encompass a cost-effectiveness and clinical outcomes assessment.

Table I Determining appropriateness for scale-up

Case study: Dr Smith and her postdoctoral student, Jane, developed an intervention to improve medication adherence for elderly patients with hypertension. Their intervention was developed after a careful review of existing scientific literature and had a sound theoretical basis (ie, KTA knowledge creation: knowledge inquiry and synthesis phases). Dr Smith presented her intervention idea to hospital leadership while designing the intervention to secure their buy-in. The intervention involved a pharmacist providing telephone-based behavioral counseling and medication management at monthly intervals (ie, KTA knowledge creation: products/tools phase). While the primary outcome was blood pressure control, Dr Smith also collected information on changes in medication adherence throughout the course of the study. Information about the length of the phone calls, cost of the intervention, and acceptability from both a pharmacist and patient perspective were also collected. The study enrolled 100 elderly patients with hypertension from a single medical center. Half of the patients were randomized to the intervention and half to their usual care. The intervention was a success. Patients in the intervention group had significant improvements in blood pressure control and medication adherence (ie, KTA action cycle: evaluate outcomes). Patients and providers alike reported that the intervention was worthwhile; however, it was not cost-effective. Dr Smith and Jane conducted additional analyses and hypothesized that, although the intervention is costly in the short-term, there would be long-term cost savings as a result of improved blood pressure control (eg, fewer heart attacks and hospitalizations). Hospital administrators were impressed with the study findings and approached Dr Smith about scaling up the intervention for delivery at two other hospitals in the health care system. They were aware that the intervention was not cost-effective, but appreciated it for its simplicity and indicated that they could fund the program for I year.

\section{Thoughts to consider}

- Has the intervention been proven beneficial to patients? Are there potential harms?

- Are the improvements in medication adherence and blood pressure control maintained over time?

- Is there new knowledge worth translating into action?

- What alterations would need to be made if the intervention is scaled up to the health care system?

Abbreviation: KTA, Knowledge to Action. 
Table 2 Allowing for flexibility in design

Case study: Dr Smith partners with hospital leadership to expand the intervention to three hospitals in the health care system. As part of this transition, it becomes part of clinical practice. Dr Smith is concerned about the fidelity to the original intervention content. The team institutes ongoing monitoring by recording telephone calls and conducting continuing education with participating pharmacists (ie, KTA action cycle: monitor and sustain knowledge use). Dr Smith identifies a problem; some pharmacists are excluding an educational component about the importance of daily home-based blood pressure monitoring. A review and brief retraining are conducted (ie, KTA action cycle: identify problem, review). During the training, several pharmacists tell Dr Smith that they are skipping this content because patients do not have tools to monitor their values at home. Dr Smith modifies the intervention content to include local information about where free blood pressure monitors are available, such as at local drugs stores (ie, KTA action cycle: adapt knowledge to local context). She talks with the intervention pharmacists to assess whether they are comfortable discussing this information with patients (ie, KTA action cycle: assess barriers to knowledge use). Over time, additional modifications are made for the local context. To reduce the cost of the intervention and save pharmacists' time, instead of providing individual telephone-based counseling the intervention is delivered to two patients at a time via a "buddy" conference call. In addition to receiving the original intervention content, patients report liking the social support aspect of the intervention.

Thoughts to consider

- Is it appropriate for Dr Smith to change the intervention content during intervention delivery?

- How could Dr Smith have included stakeholders in the ongoing monitoring and evaluation process?

- How could the intervention content or delivery be tailored for this patient population or clinical context?

Abbreviation: KTA, Knowledge to Action.

Scalable programs must be designed with a goal of simplicity. Behavioral interventions often target complex problems with multiple health determinants, necessitating multifaceted solutions. There is inevitable tension between ensuring a program is robust while balancing feasibility constraints. Complex interventions can be difficult to scale-up and sustain, whereas less complex interventions tend to be less resource intensive and easier to scale-up and sustain. Whenever possible, using simplicity in design will increase the likelihood of maintaining fidelity.

Allowances must be made for flexibility and changing course when appropriate (Table 2). One way to do this is through adaptive design, in which there are planned opportunities to evaluate a program while it is ongoing and make changes to one or more specified design aspects. ${ }^{7}$ Although improper adaptations can lead to biased studies, when used properly the benefits of adaptive design may include a smaller sample size, more efficient treatment development process, and an increased chance of correctly answering the clinical question of interest. ${ }^{7}$ Regardless of the study approach taken, gaining and maintaining stakeholder interest is another important element for ensuring sustainability. Identifying clinical and organizational leaders early on can help ensure that they share in the process and develop a sense of ownership.

\section{Selecting interventions}

Often because of time and resource constraints, it may be preferable to use an existing program rather than crafting one from scratch. In terms of the KTA Framework, this may result in limiting time spent in the knowledge creation stage and instead focusing on the action cycle. ${ }^{1}$ When choosing an existing program, it is important to consider the program match, quality, and organizational resources ${ }^{8}$ Regarding program match, it is important to gauge how well the program's goals and objectives match that of the implementing organization and its culture. ${ }^{8}$ Is the program complementary with others offered by the organization? Regarding quality, the intervention should be based on scientific evidence including robust clinical and outcomes evaluation. ${ }^{8}$ Regardless of how attractive a program may appear, an organization must

\section{Table 3 Adapting interventions}

Case study: Dr Smith's intervention is successful throughout the three-hospital health care system. Thanks to cost savings and buy-in from organizational leadership, the intervention delivery is ongoing after a year. She has published several articles describing the intervention development, implementation, and dissemination. Dr Smith's former postdoc, Jane, is now running a medication adherence clinic in another state. She reads about the success of Dr Smith's intervention in "real world" clinical practice and considers whether it would benefit patients in her clinic, but she wonders whether it is feasible in that setting. Nurses staff Jane's clinic; she does not have access to pharmacists. Also, all of the patients receiving care in Jane's clinic are given a Wi-Fi-enabled blood pressure monitor as part of their routine clinical care so providers know patients' home-monitored blood pressure values in real-time. Jane is not sure how these differences might impact the integrity of the intervention. Would it still help her patients? Dr Smith's publications do not provide any insight into whether she thinks changing the role of the interventionist will change the clinical outcome. Jane decides to contact Dr Smith and seek her insight.

Thoughts to consider

- What are the core components of Dr Smith's original intervention?

- Did the core components remain consistent as the intervention was translated from a research study (Table I) to clinical practice (Table 2)? 
Table 4 Sustaining interventions

Case study: Jane speaks with Dr Smith and is confident in her clinic's ability to adapt and implement the intervention. Jane is focused on designing and implementing the intervention so that it can be sustainable in the long-term. She immediately begins planning for the intervention implementation. She gathers relevant evidence by reviewing scientific literature, speaking with $\mathrm{Dr}$ Smith, and meeting with leaders at other sites that have implemented the intervention. Next, she seeks the commitment and support of top leaders in her organization, as well as front-line clinic staff. In doing this, Jane develops partnerships both internal and external to the clinic. She cultivates a partnership with a blood pressure monitor manufacturer and a local patient advocacy group. She also identifies program champions within her institution; there are two nurse managers who are very enthusiastic about the program. Jane wants to build capacity to carry out and sustain the intervention, so she invests in training for clinic staff in how to educate patients to use their monitors and in the intervention content. She goes a step further by having train-the-trainer sessions, so that in the future her own staff can train those who are new to the clinic. Jane plans ahead and meets with her organization's administrators. They agree that, if the intervention is successful and is cost-effective, it may be possible to embed the intervention into the organization's core policies. However, Jane must ensure that the intervention effectiveness and outcomes are evaluated on an ongoing basis. While Jane is keen on implementing the intervention and planning for sustainability, she recognizes that the program may have to evolve and adapt in order to stay viable. She plans to re-evaluate key aspects of the intervention delivery at 6-month intervals. In doing this Jane plans to develop a report, along with the program champions and key stakeholders, to secure future funding. She plans to request funding from the blood pressure monitor manufacturer that has agreed to partner with the clinic on the project, as well as a foundation that offers funds to improve patient care.

Thoughts to consider

- What additional steps could Jane take to encourage intervention sustainability?

- Are there other partnerships that Jane needs to develop?

- How could she keep key stakeholders, partners, and program champions engaged with the intervention?

- Is there a role for an advisory board or committee for sustainability?

have adequately trained and available staff, financial support, physical space, and leadership support in order for it to be successful.

\section{Adapting interventions}

It is often necessary to adapt programs for unique situations. Adaptation involves modifying an existing program to make it more suitable for a particular population or to better fit with an organization's capacity and needs without compromising its integrity. ${ }^{9}$ In the KTA Framework, this is conceptualized throughout the action cycle. For example, two phases of the action cycle are: 1) identify, review, and select knowledge; and 2) adapt knowledge to local context. ${ }^{1,4}$ Adaptation may be merited when applying a program to a new organization or community, to better fit with resources or budgetary constraints, or to better fit with local preferences or culture. ${ }^{9}$ When making adaptations, a balance must be struck between maintaining the fidelity of the original intervention while being flexible enough to meet the current needs and setting. As a starting point for successful adaptation, the core components of the original intervention must be acknowledged. Core components are essentially the active ingredients. Core components may be classified as the content of the intervention, the pedagogy of how that content is delivered, and the logistics of the implementation or delivery of the content. ${ }^{9}$ Because adaptation may apply an intervention to a more heterogeneous population, it may also increase the program's external validity (Table 3 ).

\begin{tabular}{|c|}
\hline $\begin{array}{l}\text { Plan } \\
\text { - Gather relevant evidence and consider theoretical frameworks } \\
\text { - Seek commitment and support } \\
\text { - Develop partnerships and identify program champions } \\
\text { - Think about future scale-up and implementation } \\
\text { - Consider organizational environment, cost and resource requirements } \\
\text { - Determine whether it is preferable to design an intervention or select and } \\
\text { adapt an existing one }\end{array}$ \\
\hline$\uparrow$ \\
\hline $\begin{array}{l}\text { Do } \\
\text { - Determine efficacy on a small scale }\end{array}$ \\
\hline 尔 \\
\hline $\begin{array}{l}\text { Study } \\
\text { - Evaluate whether the intervention is worth scaling up } \\
\text { - Is it safe? } \\
\text { - Is it clinically effective? } \\
\text { - Is it cost-effective? } \\
\text { - Assess the feasibility of scale-up } \\
\text { - Are the needed resources available (eg, personnel, organizational } \\
\text { resources, technological resources)? } \\
\text { - Consider contextual factors (eg, timing, organization's climate and } \\
\text { readiness to change, buy-in of key leaders) } \\
\text { - Consider the cost }\end{array}$ \\
\hline$\downarrow$ \\
\hline $\begin{array}{l}\text { Adjust } \\
\text { - Identify core components and leave those in place } \\
\text { - Balance fidelity and flexibility } \\
\text { - Simplify, adapt, and adjust }\end{array}$ \\
\hline$\downarrow$ \\
\hline $\begin{array}{l}\text { Sustain } \\
\text { - Review factors from planning stage } \\
\text { - Build capacity } \\
\text { - Embed intervention into core policies } \\
\text { - Continue evaluation of clinical and implementation outcomes } \\
\text { - Secure funding }\end{array}$ \\
\hline
\end{tabular}

Figure I Putting it all together - suggestions for sustainable programs.

Notes: The phases in Figure I need not be sequential. For example, it is possible to move from the planning phase, to the doing phase and the study phase, determine that the intervention is not cost-effective, and then cycle back to the planning phase. 


\section{Sustaining interventions}

Regardless of whether an organization is developing or identifying and/or adapting an existing program, the sustainability of the program must be evaluated. Sustainability can be thought of in several different dimensions: program sustainability over time or maintaining the improvement in outcome. Because interventions occur in complex societal systems, sustaining an intervention may require action at many levels ranging from knowledge use, to individual change, to community engagement, to institutional change. ${ }^{1,10}$ There are several elements that may predict program sustainability. Whelan et $\mathrm{a}^{11}$ assert that these elements include: planning, gathering relevant evidence, seeking commitment and support, developing partnerships, identifying program champions, building capacity, embedding into core policy, evaluating effectiveness and outcomes, evolving and adapting, and securing funding (Table 4).

\section{Conclusion}

While many effective programs are developed, relatively few successfully transition from the research setting to real world clinical practice. Using the KTA Framework as a guide, we assert that with thoughtful planning it is possible to implement and sustain interventions in clinical practice. This will require designing or selecting and adapting interventions with the potential for future scale-up. Interventions must be reasonable to implement from a resource and cost perspective. Garnishing stakeholder support and having plans for longterm funding are among the critical elements to sustaining an intervention over time (Figure 1). This is critical not only for advancing the field, but also in order to see interventions having a lasting impact on patient care.

\section{Acknowledgments}

Dr Bosworth was supported by a research career scientist award (VA HSR\&D 08-027). Dr Zullig was supported by Veterans
Affairs (VA) Health Services Research and Development (HSR\&D) Career Development Award (CDA 13-025).

\section{Disclosure}

The authors have no conflicts of interest to disclose. The views expressed in this article are those of the author(s) and do not necessarily represent the views of the Department of Veterans Affairs.

\section{References}

1. Graham ID, Logan J, Harrison MB, et al. Lost in knowledge translation: time for a map? J Contin Educ Health Prof. 2006;26(1):13-24.

2. Agency for Healthcare Research and Quality. Accelerating Change and Transformation in Organizations and Networks II. March 2013. Available from: http://www.ahrq.gov/cpi/initiatives/ACTION_II/index. html. Accessed February 5, 2015.

3. Field B, Booth A, Ilott I, Gerrish K. Using the knowledge to action framework in practice: a citation analysis and systematic review. Implement Sci. 2014;9(1):172.

4. Graham ID, Tetroe J. Some theoretical underpinnings of knowledge translation. Acad Emerg Med. 2007;14(11):936-941.

5. Milat AJ, King L, Bauman AE, Redman S. The concept of scalability: increasing the scale and potential adoption of health promotion interventions into policy and practice. Health Promot Int. 2013;28(3): 285-298.

6. Roumie CL, Arbogast PG, Mitchel EF Jr, Griffin MR. Prescriptions for chronic high-dose cyclooxygenase- 2 inhibitors are often inappropriate and potentially dangerous. J Gen Intern Med. 2005;20(10):879-883.

7. Kairalla JA, Coffey CS, Thomann MA, Muller KE. Adaptive trial designs: a review of barriers and opportunities. Trials. 2012;13:145.

8. Small S, Cooney S, Eastman G, O'Conner C. Guidelines for selecting an evidence-based program: balancing community needs, program quality, and organizational resources. What Works - Wisconsin Research to Practice Series. 2007; Number 3.

9. Family and Youth Services Bureau. Making Adaptations Tip Sheet. July 15, 2011. Available from: http://www.acf.hhs.gov/sites/default/ files/fysb/prep-making-adaptations-ts.pdf. Accessed October 8, 2014.

10. Swerissen H, Crisp BR. The sustainability of health promotion interventions for different levels of social organization. Health Promot Int. 2004;19(1):123-130.

11. Whelan J, Love P, Pettman T, et al. Cochrane update: predicting sustainability of intervention effects in public health evidence: identifying key elements to provide guidance. J Publ Health (Oxf). 2014;36(2): $347-351$.
Journal of Multidisciplinary Healthcare

\section{Publish your work in this journal}

The Journal of Multidisciplinary Healthcare is an international, peerreviewed open-access journal that aims to represent and publish research in healthcare areas delivered by practitioners of different disciplines. This includes studies and reviews conducted by multidisciplinary teams as well as research which evaluates the results or conduct of such teams or

\section{Dovepress}

healthcare processes in general. The journal covers a wide range of areas and welcomes submissions from practitioners at all levels, from all over the world. The manuscript management system is completely online and includes a very quick and fair peer-review system. Visit http://www.dovepress.com/testimonials.php to read real quotes from published authors. 